Research Article

\title{
Finite-Time Sliding Mode Control Design for Unknown Nonaffine Pure-Feedback Systems
}

\author{
Jun Zhou and Xianqiang $\mathrm{Li}$ \\ Institute of Precision Guidance and Control, Northwestern Polytechnical University, Xian 710072, China \\ Correspondence should be addressed to Xianqiang Li; jingdaosuo803@163.com
}

Received 22 April 2015; Accepted 5 August 2015

Academic Editor: Zhan Shu

Copyright ( 92015 J. Zhou and X. Li. This is an open access article distributed under the Creative Commons Attribution License, which permits unrestricted use, distribution, and reproduction in any medium, provided the original work is properly cited.

\begin{abstract}
A class of unknown nonaffine pure-feedback nonlinear systems is investigated and a novel output feedback control scheme with low complexity is proposed, based on the sliding mode control theory. The scheme is capable of guaranteeing output tracking error with finite-time convergence and bounded closed loop signals. In this scheme, a novel transformation method is included, which can easily transform the state-feedback control of nonaffine systems into output feedback control of strict-feedback affine systems. Based on the transformed affine systems, a novel finite-time sliding mode control is designed, which is continuous and nonsingular. The control scheme proposed in this work is simple and easy to implement, in which the "explosion of complexity" caused by backstepping-like scheme is completely avoided. And the finite-time convergence is successfully achieved. In addition, the scheme is designed based on output feedback control. And the dynamics of the nonaffine nonlinear systems is unknown in the design process. Thus, the system knowledge needed is reduced.
\end{abstract}

\section{Introduction}

In the past decade, control design for complex nonlinear systems has attracted a lot of attention. And many breakthrough results have been obtained, such as feedback linearization technical [1] and adaptive backstepping technical [2]. The aforementioned results assumed the input is affine in the systems considered. In practice, there are many nonlinear systems with nonaffine structure, such as aircraft dynamics [3] and mechanical systems [4]. Explicit inverting control design is impossible for nonaffine systems, because the input does not appear linearly in the systems. Therefore, the control design for nonaffine systems is more complex and more challenging.

Many researches have been done for nonaffine purefeedback nonlinear systems. In the literatures [5-10], nonaffine pure-feedback systems are studied, in which the model is known. In practice, it is difficult to obtain an exact dynamic model of the system. And in the literature [11-14], the controller design for unknown nonaffine pure-feedback systems is studied. However, the proposed control methods above are all designed based on the backstepping technical.
A drawback with the back-stepping technique is the problem of "explosion of complexity," which is caused by the repeated differentiations of certain nonlinear functions such as virtual controls $[15,16]$. To overcome the problem, dynamic surface control (DSC) was proposed in the controller design for nonaffine system [17], by employing first-order filtering of the synthetic virtual control input at each step of backstepping approach. However, this method will produce an algebraic loop, because the controller is employed in the approximation algorithm, whose output is simultaneously utilized in the controller. The problem of algebraic loop is solved in [18] by combining the DSC technique and input-to-state stability(ISS-) modular design method [6]. However, in [18] the corresponding neural network (NN) and filter are needed to be designed in each recursive step. Thus, the method is still complex. Recently, a low-complexity global approximation-free backstepping-like approach is proposed for pure-feedback nonaffine systems in [19], under the assumption that the full states are known. And the complexity explosion issue is avoided. However, it is still a recursive method. And it is usually impossible that all the states are available in the actual process. Hence, for nonaffine systems, it needs to 
further study the low-complexity controller design that is not based on the backstepping technique and needs less system knowledge.

It has been recognized that sliding mode control is efficient to design a robust controller. In most of the sliding mode controllers, the sliding mode surface is linear, which can only guarantee the infinite-time convergence of the states $[20,21]$. To implement finite-time convergence, nonlinear sliding mode surfaces are employed. One of such sliding mode surfaces is the terminal sliding mode (TSM) [22]. However, TSM controller may cause the singularity problem in the closed-loop system [23]. To overcome the singularity problem, the nonsingular terminal sliding mode control (NTSM) is proposed in $[24,25]$. However, NTSM can only be used in the second-order system. For high-order systems, a finite-time sliding mode controller is designed in [26]. However, the sliding mode controllers mentioned above are all designed for affine systems and are difficult to directly be applied to nonaffine systems. According to the authors' best knowledge, all the sliding mode control designed for nonaffine systems [27-32] can only guarantee the infinitetime convergence of the states. The finite-time controllers have faster convergence rate than that of infinite-time controllers. Furthermore, they possess better robustness and better disturbance rejection performance, because fractional power items are contained in them $[33,34]$. Thus, research on finite-time control of nonaffine systems has important practical and theoretical significance.

In this paper, a novel finite-time convergence control scheme is proposed for unknown nonaffine pure-feedback system, based on sliding mode control theory. In the design procedure, the input of the system is augmented with a lowpass filter firstly. Then the nonaffine system is transformed into an affine strict-feedback system through states transformation. Because the states in the transformed system are unavailable for controller design, a finite-time differentiator is introduced to obtain the unavailable states. Finally, a finitetime sliding mode controller is designed. Main contributions of the paper are the following:

(1) Complexity of the control for nonaffine system is reduced dramatically. A novel transformation method is proposed, capable of completely avoiding "explosion of complexity" caused by backsteppinglike scheme. Moreover, in the proposed scheme, only one estimator is needed to obtain the states used in the controller.

(2) The finite-time convergence of the closed system is achieved for the first time. For nonaffine purefeedback nonlinear systems, a nonsingular, finitetime sliding mode control is designed for the first time. And the controller is continuous; thus, the chattering of actuators is alleviated.

(3) The system knowledge needed is reduced. In the proposed scheme, only the outputs of the system, other than all the states, are used. Furthermore, the control is designed based on the unknown nonaffine pure-feedback systems; thus, accurate model of the system is not required.

\section{Model and Problem Formulation}

Consider a class of nonaffine pure-feedback systems as follows:

$$
\begin{aligned}
\dot{x}_{i}(t) & =f_{i}\left(x_{1}, \ldots, x_{i}, x_{i+1}\right), \quad i=1, \ldots, n-1, \\
\dot{x}_{n}(t) & =f_{n}\left(x_{1}, \ldots, x_{n}, u\right), \\
y & =x_{1}
\end{aligned}
$$

in which $x_{i}(t) \in \Re, i=1, \ldots, n$, are the states, $u \in \Re$ is the input, $y$ denotes the output, and $f_{i} \in \mathfrak{R}, i=1, \ldots, n$, are unknown smooth nonlinear functions.

Assumption 1. In (1), $f_{i}, i=1, \ldots, n$, are continuous and differentiable, satisfying

$$
\begin{aligned}
& b_{i 0} \leq \frac{\partial f_{i}\left(x_{1}, \ldots, x_{i}, x_{i+1}\right)}{\partial x_{i+1}} \leq b_{i 1}, \quad i=1, \ldots, n-1, \\
& b_{n 0} \leq \frac{\partial f_{n}\left(x_{1}, \ldots, x_{n}, u\right)}{\partial u} \leq b_{n 1}
\end{aligned}
$$

in which $b_{i 0}$ and $b_{i 1}, i=1, \ldots, n$, are positive constant value (i.e., $b_{i 0}>0, b_{i 1}>0, i=1, \ldots, n$ ).

Assumption 1 is a commonly used sufficient condition for controllability of nonaffine system [19].

Assumption 2. The desired trajectory $y_{d}: \mathfrak{R}_{+} \rightarrow \mathfrak{R}$ is known. It is sufficiently smooth, and there are constants $c_{i}, i=1, \ldots, n+1$, satisfying $\left|y_{d}^{(i)}\right| \leq c_{i}$.

In this paper, we focus on the control of unknown nonaffine pure-feedback system. The objective is to obtain a low-complexity controller, such that the tracking error with respect to the desired trajectory can converge to the equilibrium point with finite time, and all the states are bounded.

\section{Finite-Time Output Feedback Control Based on Sliding Mode Control Theory}

3.1. Design of a Novel System Transformation Method. Recently, in [21], a low-pass filter used to attenuate the chattering is introduced in the input channel (i.e., $\dot{u}=T u+v$ ), when designing the sliding mode control for affine systems. Inspired by this, we proposed a novel transformation method, in which we will show that the low-pass filter can also be used to transform the nonaffine systems into affine systems. The transformation procedure is given as follows.

Augment the nonaffine system (1) through introducing a low-pass filter in the input channel. And the augmented system can be denoted by the following:

$$
\begin{aligned}
\dot{x}_{i}(t) & =f_{i}\left(x_{1}, \ldots, x_{i}, x_{i+1}\right), \quad i=1, \ldots, n-1, \\
\dot{x}_{n}(t) & =f_{n}\left(x_{1}, \ldots, x_{n}, u\right), \\
\dot{u} & =T u+v, \\
y & =x_{1} .
\end{aligned}
$$


In (3), $T>0, u$ becomes a new state, and $v$ denotes the new control input.

Let $e_{1}=x_{1}-y_{d}$. And definite new states $\left[\begin{array}{llll}q_{1} & q_{2} & \cdots & q_{n+1}\end{array}\right]^{T}$, in which $q_{1}=e_{1}, q_{i}=e^{(i-1)}, i=$ $2, \ldots, n+1$. Based on (3), one can obtain

$$
\begin{aligned}
\dot{q}_{1} & =\dot{x}_{1}-\dot{y}_{d}, \\
\dot{q}_{i+1} & =f_{1}^{(i)}-y_{d}^{(i+1)}=\sum_{j=1}^{i+1} \frac{\partial q_{i+1}}{\partial x_{j}} f_{j}-y_{d}^{(i+1)}, \\
\dot{q}_{n+1} & =f_{1}^{(n)}-y_{d}^{(n+1)} \\
& =\sum_{j=1}^{n} \frac{\partial q_{n+1}}{\partial x_{j}} f_{j}+\frac{\partial q_{n+1}}{\partial u}(T u+v)-y_{d}^{(n+1)} .
\end{aligned}
$$

Now, the initial nonaffine system denoted by (1) is transformed into an affine strict-feedback integral chain system denoted by the following:

$$
\begin{aligned}
\dot{q}_{i} & =q_{i+1}, \quad i=1, \ldots, n, \\
\dot{q}_{n+1} & =F+B v
\end{aligned}
$$

in which

$$
\begin{aligned}
& F=\sum_{j=1}^{n} \frac{\partial q_{n+1}}{\partial x_{j}} f_{j}+\frac{\partial q_{n+1}}{\partial u} T u-y_{d}^{(n+1)}, \\
& B=\frac{\partial q_{n+1}}{\partial u}=\frac{\partial f_{n}}{\partial u} \prod_{i=1}^{n-1} \frac{\partial f_{i}}{\partial x_{i+1}} .
\end{aligned}
$$

Base on Assumption 1, one can obtain $0<\prod_{i=1}^{n} b_{i 0} \leq$ $B \leq \prod_{i=1}^{n} b_{i 1}$. Moreover, if $|F| \leq \omega$, combing (5) and (6), the following differential inclusion can be concluded:

$$
\dot{q}_{n+1} \in[-\omega, \omega]+\left[K_{m}, K_{M}\right] v
$$

in which $K_{m}=\prod_{i=1}^{n} b_{i 0}, K_{M}=\prod_{i=1}^{n} b_{i 1}$.

3.2. Design of a Finite-Time Differentiator. In transformed system (5), only the states $q_{1}=e_{1}$ are measurable, and the states $q_{i}, i=2, \ldots, n+1$, are not available. All the unmeasurable states can be obtained through solving the derivatives of $q_{1}$. In this section, a finite-time differentiator is given by

$$
\begin{gathered}
\dot{\varsigma}_{1}=v_{1}=-\lambda_{n+1} L^{1 /(n+1)}\left|\varsigma_{1}-q_{1}\right|^{n /(n+1)} \operatorname{sign}\left(\varsigma_{1}-q_{1}\right) \\
+\varsigma_{2} \\
\vdots \\
\dot{\zeta}_{n}=v_{n}=-\lambda_{2} L^{1 / 2}\left|\varsigma_{n}-v_{n-1}\right|^{1 / 2} \operatorname{sign}\left(\varsigma_{n}-v_{n-1}\right)+\varsigma_{n+1} \\
\dot{\zeta}_{n+1}=v_{n+1}=-\lambda_{1} L \operatorname{sign}\left(\varsigma_{n+1}-v_{n}\right),
\end{gathered}
$$

where $L$ is a Lipschitz constant of $q_{1}^{(n+1)}$ and $\varsigma_{i}$ is the estimate of $q_{1}^{(i-1)}$.

Define the estimated errors and their derivatives as $e_{i}=$ $\varsigma_{i}-q_{1}^{(i-1)}=\varsigma_{i}-q_{i}, i=1, \ldots, n+1$. The following dynamic equations can be obtained:

$$
\begin{gathered}
\dot{e}_{1}=-\lambda_{n} L^{1 /(n+1)}\left|e_{1}\right|^{n /(n+1)} \operatorname{sign}\left(e_{1}\right)+e_{2} \\
\vdots \\
\dot{e}_{n}=-\lambda_{1} L^{1 / 2}\left|e_{n}-\dot{e}_{n-1}\right|^{1 / 2} \operatorname{sign}\left(e_{n}-\dot{e}_{n-1}\right)+e_{n} \\
\dot{e}_{n+1} \in-\lambda_{0} L \operatorname{sign}\left(e_{n+1}-\dot{e}_{n}\right)+[-L, L] .
\end{gathered}
$$

It follows from [35] that the estimated errors can converge to zero in finite time.

3.3. Design of a Nonsingular Finite-Time Sliding Mode Control. A nonlinear sliding mode surface is given by

$$
s=\varsigma_{n+1}+\int_{0}^{t}\left[\sum_{i=1}^{n+1} k_{i} \operatorname{sign}\left(\varsigma_{i}\right)\left|\varsigma_{i}\right|^{\alpha_{i}}\right] d t
$$

in which $k_{i}, i=1, \ldots, n+1$, can ensure that the polynomial $p^{n+1}+k_{n+1} p^{n}+\cdots+k_{2} p+k_{1}=0$ is Hurwitz, $\alpha_{i} \in(0,1), i=$ $1, \ldots, n+1$, satisfying $\alpha_{i-1}=\alpha_{i} \alpha_{i+1} /\left(2 \alpha_{i+1}-\alpha_{i}\right), i=2, \ldots, n+$ $1, \alpha_{n+2}=1, \alpha_{n+1}=\alpha_{0} \in(0,1)$.

Theorem 3. Consider nonaffine pure-feedback system (1), which satisfies Assumptions 1 and 2. All the initial states $\left(x_{1}\left(t_{0}\right), x_{2}\left(t_{0}\right), \ldots, x_{n}\left(t_{0}\right)\right) \in \varphi_{0}$ and $\varphi_{0} \in \mathfrak{R}^{n}$ is a compact set. When choosing the control input denoted by

$$
\begin{aligned}
& \dot{u}=T u+v, \\
& v=v_{1}+v_{2}, \\
& v_{1}=-B_{0}^{-1} \sum_{i=1}^{n+1} k_{i} \operatorname{sign}\left(\varsigma_{i}\right)\left|\varsigma_{i}\right|^{\alpha_{i}}, \\
& v_{2}=-B_{0}^{-1} \lambda s-B_{0}^{-1} \psi \operatorname{sign}(s), \\
& \psi=\left(\eta+B_{0}\left(\rho+\varepsilon_{1}\left|v_{1}\right|\right)\right)\left(1-\varepsilon_{0}\right)^{-1}
\end{aligned}
$$

in which the sliding mode surface $s$ is designed as (10), and $\varsigma_{i},(i=0,1, \ldots, n)$, are obtained by differentiator (8), $T>$ $0, B_{0}>0$, satisfying $\prod_{i=1}^{n} b_{i 1}-B_{0}>B_{0}-\prod_{i=1}^{n} b_{i 0}>0,0<\varepsilon_{0} \leq$ $B_{0}^{-1}\left(B_{0}-\prod_{i=1}^{n} b_{i 0}\right), \lambda>0, \varepsilon_{1} \geq B_{0}^{-1}\left(\prod_{i=1}^{n} b_{i 1}-B_{0}\right), \eta>0, \rho$, satisfying $B_{0} \rho>F$, then the output tracking error $q_{1}=x_{1}-y_{d}$ will converge to zero in finite time, and all the signals in the close-loop system are bounded. 
Proof. According to $e_{i}=\varsigma_{i}-q_{1}^{(i-1)}=\varsigma_{i}-q_{i}, i=1, \ldots, n+1$, one can obtain $\zeta_{n+1}=q_{n+1}+e_{n+1}$. Then combing (5) and (10) yields

$$
\begin{aligned}
\dot{s} & =\dot{\varsigma}_{n+1}+\sum_{i=1}^{n+1} k_{i} \operatorname{sign}\left(\varsigma_{i}\right)\left|\varsigma_{i}\right|^{\alpha_{i}} \\
& =F+B v+\dot{e}_{n+1}+\sum_{i=1}^{n+1} k_{i} \operatorname{sign}\left(\varsigma_{i}\right)\left|\varsigma_{i}\right|^{\alpha_{i}} \\
& =F+B_{0}(1+\varepsilon) v+\dot{e}_{n+1}+\sum_{i=1}^{n+1} k_{i} \operatorname{sign}\left(\varsigma_{i}\right)\left|\varsigma_{i}\right|^{\alpha_{i}} .
\end{aligned}
$$

Because $B_{0}>0$, satisfying $\prod_{i=1}^{n} b_{i 1}-B_{0}>B_{0}-\prod_{i=1}^{n} b_{i 0}>0,0<$ $\varepsilon_{0} \leq B_{0}^{-1}\left(B_{0}-\prod_{i=1}^{n} b_{i 0}\right), \varepsilon_{1} \geq B_{0}^{-1}\left(\prod_{i=1}^{n} b_{i 1}-B_{0}\right)$, it implies that $\varepsilon \in\left[-\varepsilon_{0}, \varepsilon_{1}\right], 0<\varepsilon_{0}<1, \varepsilon_{1}>0, \varepsilon_{1}>\varepsilon_{0}$.

Noting $e_{i}=\varsigma_{i}-q_{1}^{(i-1)}=\varsigma_{i}-q_{i}, i=1, \ldots, n+1$, and combing (10), the dynamic of $\varsigma_{i},(i=1, \ldots, n+1)$ can be obtained as follows:

$$
\begin{aligned}
\dot{\zeta}_{i} & =\varsigma_{i+1}+\widetilde{e}_{i}, \quad i=1, \ldots, n, \\
\dot{\zeta}_{n+1} & =\dot{s}-\sum_{i=1}^{n+1} k_{i} \operatorname{sign}\left(\varsigma_{i}\right)\left|\varsigma_{i}\right|^{\alpha_{i}},
\end{aligned}
$$

where $\widetilde{e}_{i}=\dot{e}_{i}-e_{i+1}, i=1, \ldots, n$.

It denotes that dynamic (12) and dynamic (13) are affected by the estimated error dynamic (9). In the following, it will show that dynamic (12) and dynamic (13) will not be driven into infinity in finite time by dynamic (9).

Define a finite-time bounded function as follows:

$$
V=\frac{1}{2} \sum_{i=1}^{n+1} \varsigma_{i}^{2}+\frac{1}{2} s^{2} .
$$

Taking derivative of $V$ with respect to time, then substituting (13), yields

$$
\begin{aligned}
\dot{V}= & \sum_{i=1}^{n} \varsigma_{i}\left(\varsigma_{i+1}+\widetilde{e}_{i}\right)+\varsigma_{n+1}\left(\dot{s}-\sum_{i=1}^{n+1} k_{i} \operatorname{sign}\left(\varsigma_{i}\right)\left|\varsigma_{i}\right|^{\alpha_{i}}\right) \\
& +s \dot{s} .
\end{aligned}
$$

Noting that when $\alpha_{i} \in(0,1)(i=1, \ldots, n+1)$, it implies that $\left|\varsigma_{i}\right|^{\alpha_{i}} \leq 1+\left|\varsigma_{i}\right|$, and combing (15), one can obtain

$$
\begin{aligned}
\dot{V} \leq & \sum_{i=1}^{n}\left|\varsigma_{i}\right|\left(\left|\varsigma_{i+1}\right|+\left|\widetilde{e}_{i}\right|\right) \\
& +\left|\varsigma_{n+1}\right|\left(|\dot{s}|+\sum_{i=1}^{n+1} k_{i}\left(1+\left|\varsigma_{i}\right|\right)\right)+|s||\dot{s}| \\
\leq & \frac{1}{2} \sum_{i=1}^{n}\left(\varsigma_{i}^{2}+\varsigma_{i+1}^{2}\right)+\frac{1}{2} \sum_{i=1}^{n}\left(\varsigma_{i}^{2}+\widetilde{e}_{i}^{2}\right)+\left|\varsigma_{n+1}\right||\dot{s}| \\
& +\frac{1}{2} \varsigma_{n+1}^{2}+\frac{1}{2}\left(\sum_{i=1}^{n+1} k_{i}\right)^{2}+\frac{1}{2} \sum_{i=1}^{n+1} k_{i}\left(\varsigma_{n+1}^{2}+\varsigma_{i}^{2}\right) \\
& +|s||\dot{s}| \leq l_{1} V+l_{2}+\left(\left|\varsigma_{n+1}\right|+|s|\right)|\dot{s}|
\end{aligned}
$$

in which

$$
\begin{aligned}
& l_{1}=3+2\left(\sum_{i=1}^{n+1} k_{i}\right), \\
& l_{2}=\sum_{i=1}^{n} \frac{\widetilde{e}_{i}^{2}}{2}+\frac{1}{2}\left(\sum_{i=1}^{n+1} k_{i}\right)^{2} .
\end{aligned}
$$

Substituting (11) into (12) yields

$$
\begin{aligned}
\dot{s}= & -\left(1-\varepsilon_{0}\right)^{-1}(1+\varepsilon) \\
& \cdot\left(\eta+B_{0} \rho+\left.\varepsilon_{1}\left|\sum_{i=1}^{n+1} k_{i}\right| \varsigma_{i}\right|^{\alpha_{i}} \operatorname{sign}\left(\varsigma_{i}\right) \mid\right) \operatorname{sign}(s) \\
& -\varepsilon \sum_{i=1}^{n+1} k_{i} \operatorname{sign}\left(\varsigma_{i}\right)\left|\varsigma_{i}\right|^{\alpha_{i}}-(1+\varepsilon) \lambda s+F+\dot{e}_{n+1} .
\end{aligned}
$$

Note that $\varepsilon \in\left[-\varepsilon_{0}, \varepsilon_{1}\right], 0<\varepsilon_{0}<1, \varepsilon_{1}>\varepsilon_{0}>0$, and $|F|<B_{0} \rho$ and $\left|\varsigma_{i}\right|^{\alpha_{i}} \leq 1+\left|\varsigma_{i}\right|$. And from (18), one can obtain

$|\dot{s}|$

$$
\begin{aligned}
\leq & \left(1-\varepsilon_{0}\right)^{-1}(1+\varepsilon)\left(\eta+B_{0} \rho+\varepsilon_{1} \sum_{i=1}^{n+1} k_{i}\left|\varsigma_{i}\right|^{\alpha_{i}}\right) \\
& +\varepsilon \sum_{i=1}^{n+1} k_{i}\left|\varsigma_{i}\right|^{\alpha_{i}}+(1+\varepsilon) \lambda|s|+|F|+\left|\dot{e}_{n+1}\right| \\
\leq & \left(1-\varepsilon_{0}\right)^{-1}\left(1+\varepsilon_{1}\right)\left(\eta+B_{0} \rho+\varepsilon_{1} \sum_{i=1}^{n+1} k_{i}\left(1+\left|\varsigma_{i}\right|\right)\right) \\
& +\varepsilon_{1} \sum_{i=1}^{n+1} k_{i}\left(1+\left|\varsigma_{i}\right|\right)+\left(1+\varepsilon_{1}\right) \lambda|s|+B_{0} \rho+\left|\dot{e}_{n+1}\right| \\
\leq & \vartheta_{1}+\vartheta_{2} \sum_{i=1}^{n+1} k_{i}\left|\varsigma_{i}\right|+\left(1+\varepsilon_{1}\right) \lambda|s| .
\end{aligned}
$$

In (19),

$$
\begin{aligned}
\vartheta_{1}= & \left(1-\varepsilon_{0}\right)^{-1}\left(1+\varepsilon_{1}\right)\left(\eta+B_{0} \rho\right) \\
& +\frac{\left(2+\varepsilon_{1}-\varepsilon_{0}\right)}{\left(1-\varepsilon_{0}\right)} \varepsilon_{1}\left(\sum_{i=1}^{n+1} k_{i}\right)+B_{0} \rho+\left|\dot{e}_{n+1}\right|, \\
\vartheta_{2}= & \frac{\left(2+\varepsilon_{1}-\varepsilon_{0}\right)}{\left(1-\varepsilon_{0}\right)} \varepsilon_{1}>0 .
\end{aligned}
$$


Substituting (19) into (16) yields

$$
\begin{aligned}
\dot{V} \leq & l_{1} V+l_{2}+\left(\left|\varsigma_{n+1}\right|+|s|\right)\left(\vartheta_{1}+\vartheta_{2} \sum_{i=1}^{n+1} k_{i}\left|\varsigma_{i}\right|\right) \\
& +\left(\left|\varsigma_{n+1}\right|+|s|\right)\left(1+\varepsilon_{1}\right) \lambda|s| \\
\leq & l_{1} V+l_{2}+\left(\left|\varsigma_{n+1}\right| \vartheta_{1}+|s| \vartheta_{1}\right) \\
& +\vartheta_{2} \sum_{i=1}^{n+1} k_{i}\left(\left|\varsigma_{i}\right|\left|\varsigma_{n+1}\right|+\left|\varsigma_{i}\right||s|\right) \\
& +\left(1+\varepsilon_{1}\right) \lambda \frac{\varsigma_{n+1}^{2}+3 s^{2}}{2} \\
\leq & l_{1} V+l_{2}+\vartheta_{1}^{2}+\frac{\varsigma_{n+1}^{2}+s^{2}}{2} \\
& +\vartheta_{2}\left(\sum_{i=1}^{n+1} k_{i} \frac{\varsigma_{n+1}^{2}+2 \varsigma_{i}^{2}+s^{2}}{2}\right) \\
& +\left(1+\varepsilon_{1}\right) \lambda \frac{\varsigma_{n+1}^{2}+3 s^{2}}{2} \leq\left(l_{1}+l_{3}\right) V+\left(l_{2}+l_{4}\right) .
\end{aligned}
$$

Noting (21) and (17), one can obtain

$$
\begin{aligned}
& l_{1}+l_{3}=4+\left(2+3 \vartheta_{2}\right)\left(\sum_{i=1}^{n+1} k_{i}\right)+3\left(1+\varepsilon_{1}\right) \lambda, \\
& l_{2}+l_{4}=\vartheta_{1}^{2}+\sum_{i=1}^{n} \frac{\widetilde{e}_{i}^{2}}{2}+\frac{1}{2}\left(\sum_{i=1}^{n+1} k_{i}\right)^{2},
\end{aligned}
$$

where $e_{i}, i=1, \ldots, n+1$, in (9) can converge to zero in finite time; thus, $\dot{e}_{n+1}$ and $\tilde{e}_{i}, i=1, \ldots, n$, are bounded. Consequently, $\vartheta_{1}$ and $\vartheta_{2}$ denoted by (20) are bounded. And $l_{1}+l_{3}$ and $l_{2}+l_{4}$ in (22) are bounded. Thus, it can be concluded from (21) and (14) that $V, \varsigma_{i}(i=1, \ldots, n+1)$ and $s$ will not escape to infinity in finite time before $\widetilde{e}_{i}, i=1, \ldots, n$, converge to zero.

Because $\widetilde{e}_{i}, i=1, \ldots, n$, will converge to zero in finite time, system (12) will then reduce to

$$
\dot{s}=F+B_{0}(1+\varepsilon) v+\sum_{i=1}^{n+1} k_{i} \operatorname{sign}\left(\varsigma_{i}\right)\left|\varsigma_{i}\right|^{\alpha_{i}} .
$$

Substituting (11) into (23) yields

$$
\dot{s}=B_{0} \varepsilon v_{1}+B_{0}(1+\varepsilon) v_{2}+F
$$

Noting that $\varepsilon \in\left[-\varepsilon_{0}, \varepsilon_{1}\right], \varepsilon_{1}>\varepsilon_{0}, 0<\varepsilon_{0}<1, \varepsilon_{1}>\varepsilon_{0}>0$ and $\lambda>0$, and further substituting (11) into (24), it follows that

$$
\begin{aligned}
s \dot{s} & =s B_{0} \varepsilon v_{1}+s B_{0}(1+\varepsilon) v_{2}+s F \\
& \leq B_{0}|s||\varepsilon|\left|v_{1}\right|-|s|(1+\varepsilon) \psi-(1+\varepsilon) \lambda s^{2}+|s||F| \\
& \leq B_{0} \varepsilon_{1}|s|\left|v_{1}\right|-|s|\left(1-\varepsilon_{0}\right) \psi+|s||F| .
\end{aligned}
$$

Substituting $\psi$ denoted by (11) into (25), and combing with $B_{0} \rho>F$, yields

$$
\begin{aligned}
s \dot{s} & \leq B_{0} \varepsilon_{1}|s|\left|v_{1}\right|-|s|\left(\eta+B_{0} \rho+B_{0} \varepsilon_{1}\left|v_{1}\right|\right)+|s||F| \\
& \leq-\eta|s|-|s|\left(B_{0} \rho-|F|\right) \leq-\eta|s| .
\end{aligned}
$$

According to (26), we know that the sliding mode surfaces $s$ and $\dot{s}$ will converge to zero in finite time. And (9) implies that $\widetilde{e}_{i}$ will converge to zero with finite time. So dynamic (13) will reduce to

$$
\begin{gathered}
\dot{\varsigma}_{i}=\varsigma_{i+1}, \quad i=1, \ldots, n, \\
\dot{\varsigma}_{n+1}=-\sum_{i=1}^{n+1} k_{i} \operatorname{sign}\left(\varsigma_{i}\right)\left|\varsigma_{i}\right|^{\alpha_{i}} .
\end{gathered}
$$

From (27), it can be concluded that $\varsigma_{i}(i=1, \ldots, n+1)$ and $\dot{\varsigma}_{n+1}$ will converge to zero in finite time [21]. And at this time $q_{i}=\varsigma_{i}(i=1, \ldots, n+1)$ denote the tracking error and its derivatives, so the tracking error and its derivatives are bounded and can converge to zero in finite time. Because $\varsigma_{i}, i=1, \ldots, n+1$, are all bounded, the new input $v$ in (11) is bounded. And according to the bounded input and bounded output properties of one-order linear system [36], $u$ produced by $\dot{u}=T u+v$ in (11) is bounded too. Because $q_{1}=x_{1}-y_{d}$ is bounded, state $x_{1}$ is bounded. Furthermore, because $q_{2}=f_{1}-\dot{y}_{d}$ is bounded, thus $f_{1}$ is bounded. Then it can be concluded that $x_{2}$ is bounded according to the fact that $\partial f_{1} / \partial x_{2}$ is bounded. Through using the procedure recursively, it can be concluded that all the other states $x_{i}, i=2, \ldots, n$, are bounded too.

Remark 4. Compared with the commonly used backstepping-like controller, the complexity is reduced dramatically in the proposed controller through using a novel system transformation. And the finite-time convergence of the tracking error can also be insured. The system knowledge needed is reduced, and only the output state is used in the proposed controller, while all the states are usually needed in the backstepping-like controller.

\section{Simulation}

In this section, the feasibility of the scheme proposed in this paper will be demonstrated. Consider the following threeorder system:

$$
\begin{aligned}
\dot{x}_{1}= & 0.2 x_{1}+\left(1+\exp \left(-x_{1}\right)\right) x_{2}+0.3 \sin x_{1} \\
& +0.1 \sin t \\
\dot{x}_{2}= & \frac{1-\exp \left(x_{1} x_{2}\right)}{1+\exp \left(x_{1} x_{2}\right)}+\left(1+0.2 \sin x_{1}\right) x_{3} \\
& +0.02 \sin \left(x_{2} t\right), \\
\dot{x}_{3}= & 0.5 \sin \left(x_{1} x_{2} x_{3}\right)+\left(0.42+0.1 \cos \left(x_{2} t\right)\right) u \\
& +0.1 \sin (0.5 u), \\
y= & x_{1}
\end{aligned}
$$




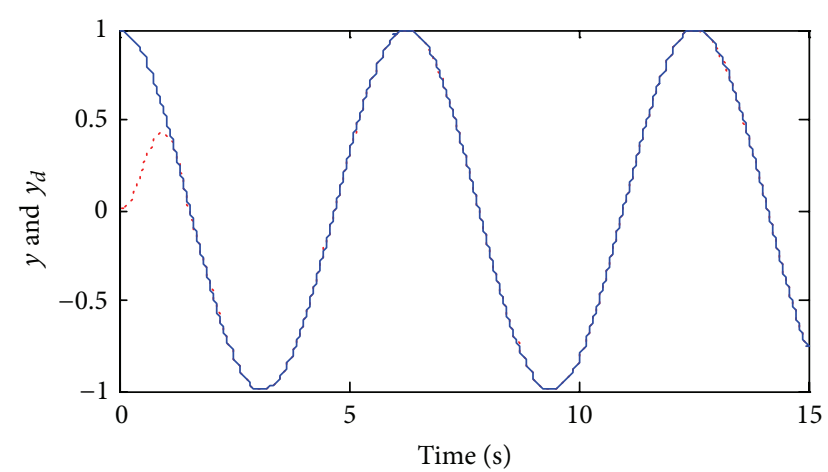

$\cdots \cdots \cdot y$

$-y_{d}$

FIGURE 1: Actual output $y$ and desired output $y_{d}$.

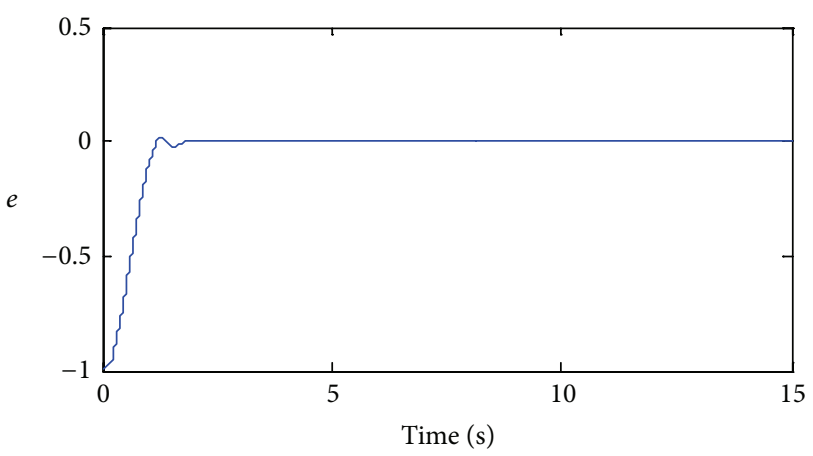

Figure 2: Tracking error.

with the desired trajectory $y_{d}=\sin (t+\pi / 2)$ and initial conditions $x_{1}(0)=0, x_{2}(0)=0, x_{3}(0)=0$.

It can be verified that Assumptions 1 and 2 are satisfied. In the controller design, the differentiator is firstly designed as (8) with the parameters: $L=300, \lambda_{0}=1.1, \lambda_{1}=30, \lambda_{2}=50$, and $\lambda_{2}=80$. The sliding mode surface is given by (10) with $k_{1}=625, k_{2}=500, k_{3}=150, k_{4}=40, \alpha_{1}=1 / 2, \alpha_{2}=$ $4 / 7, \alpha_{3}=2 / 3, \alpha_{4}=4 / 5$. And the initial value of the integral part is 0 . The controller is given by (11); in (11), $T=-5, \lambda=$ 20, $B_{0}=0.6, \eta=1, \rho=3, \varepsilon_{1}=6, \varepsilon_{0}=0.8$. The simulation results are shown as in Figures 1-10.

As a comparison, an improved backstepping-like controller designed in [18] is also simulated. In [18] the complexity issue is alleviated through using the dynamic surface technique, which can avoid calculating the derivative of the virtual control. The controller is designed as $u=-k_{3} s_{3}+$ $\widehat{\theta}_{3}^{T} \xi_{3}\left(x_{1}, x_{2}, x_{3}, \dot{z}_{3}\right)$, in which $s_{3}=x_{3}-z_{3}$ and $z_{3}$ is tuned by one-order filter $\tau_{2} \dot{z}_{3}+z_{3}=\alpha_{3} . \alpha_{3}=-k_{2} s_{2}+\widehat{\theta}_{2}^{T} \xi_{2}\left(x_{1}, x_{2}, \dot{z}_{2}\right)$ is a virtual control, in which $s_{2}=x_{2}-z_{2}$, and $z_{2}$ is tuned by one-order filter $\tau_{2} \dot{z}_{2}+z_{2}=\alpha_{2} \cdot \alpha_{2}=-k_{1} s_{1}+\widehat{\theta}_{1}^{T} \xi_{1}\left(x_{1}, \dot{y}_{d}\right)$ is a virtual control, in which $s_{1}=x_{1}-y_{d} . \theta_{i}^{T} \xi_{i}, i=1,2,3$, are three $\mathrm{NN}$ units. And the $\mathrm{NN}$ weights are determined as $\dot{\hat{\theta}}_{i}=\Gamma_{i}\left[-\xi_{i} s_{i}-\eta_{i} \widehat{\theta}_{i}\right], i=1,2,3$. The comparison of the two methods is illustrated in Figures 11-12.

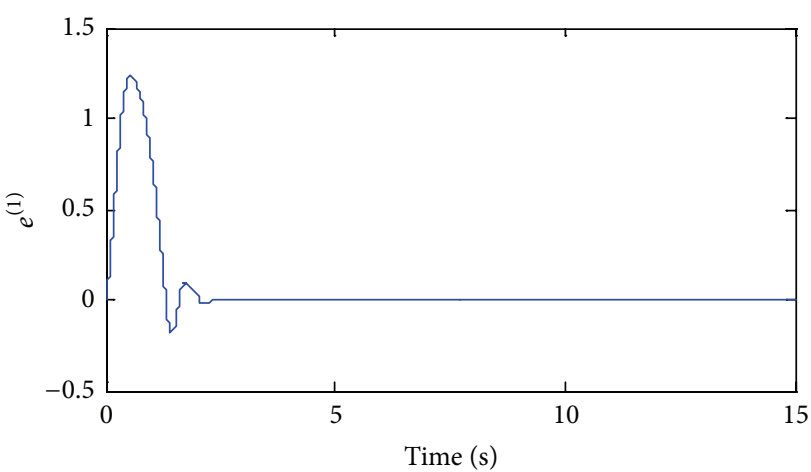

FIgURE 3: The first-order derivative of tracking error.

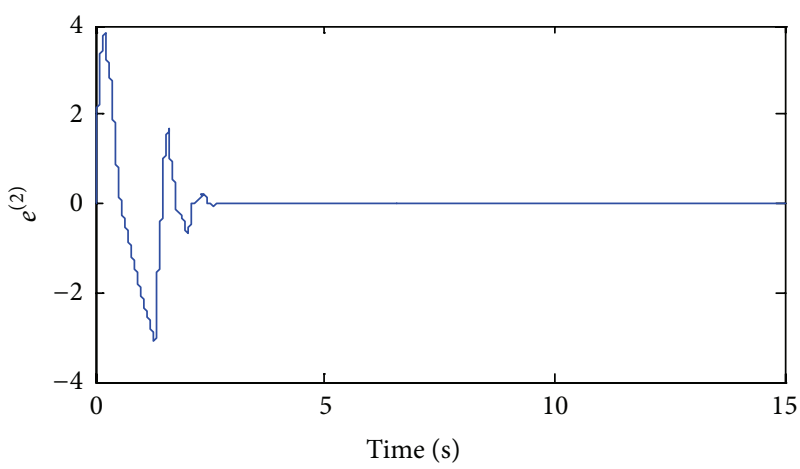

FIGURE 4: The second-order derivative of tracking error.

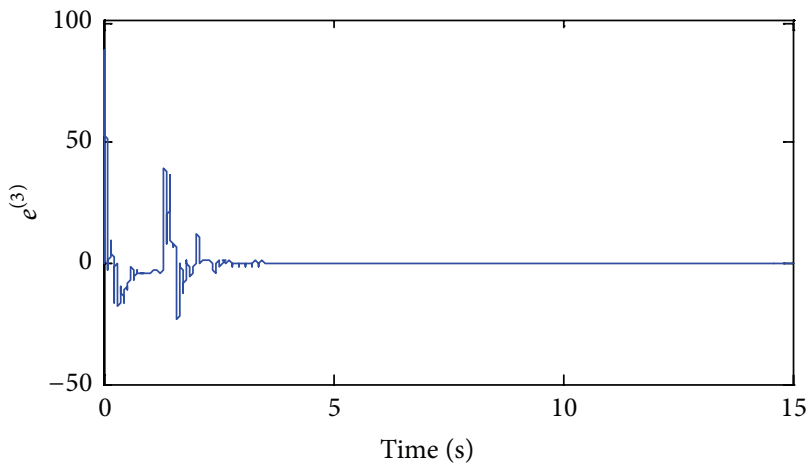

FIGURE 5: The third-order derivative of tracking error.

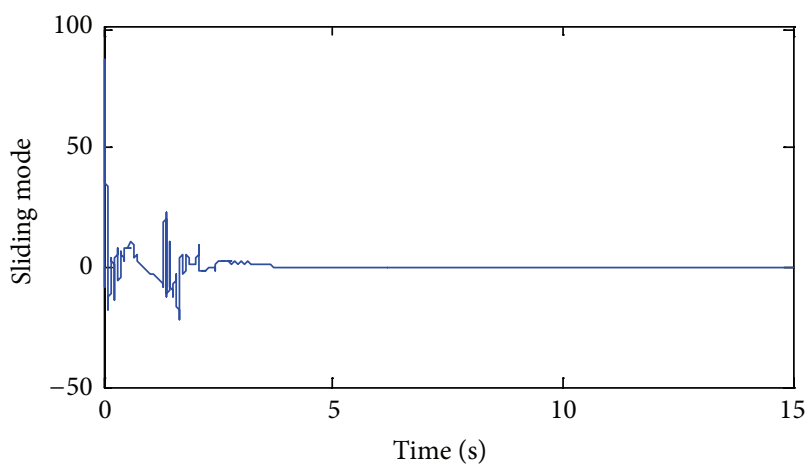

FIGURE 6: The sliding mode surface. 


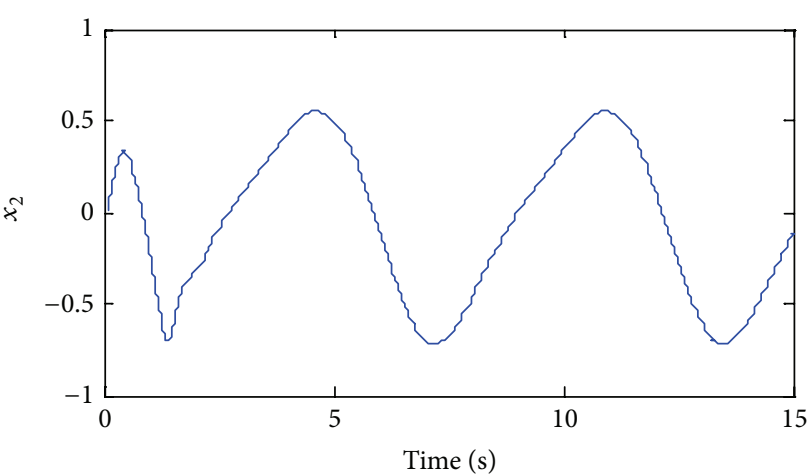

Figure 7: The closed loop system state $x_{2}$.

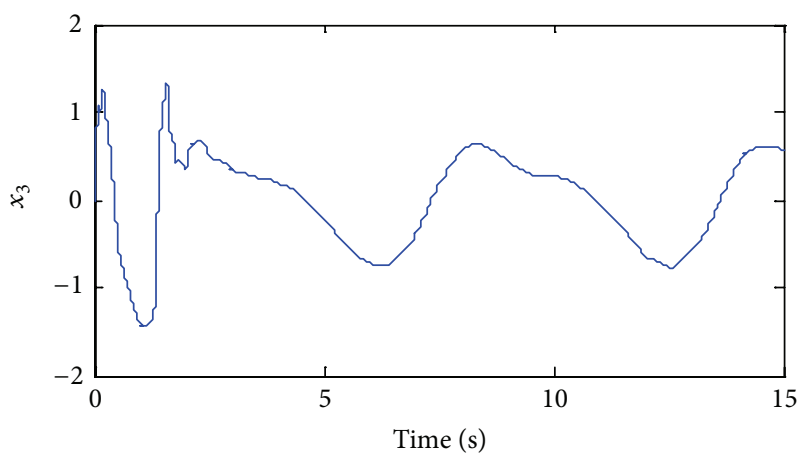

Figure 8: The closed loop system state $x_{3}$.

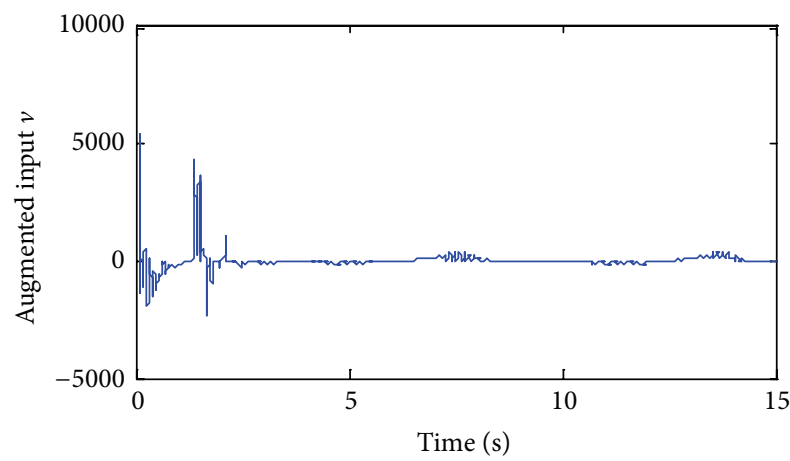

Figure 9: Augmented input $v$.

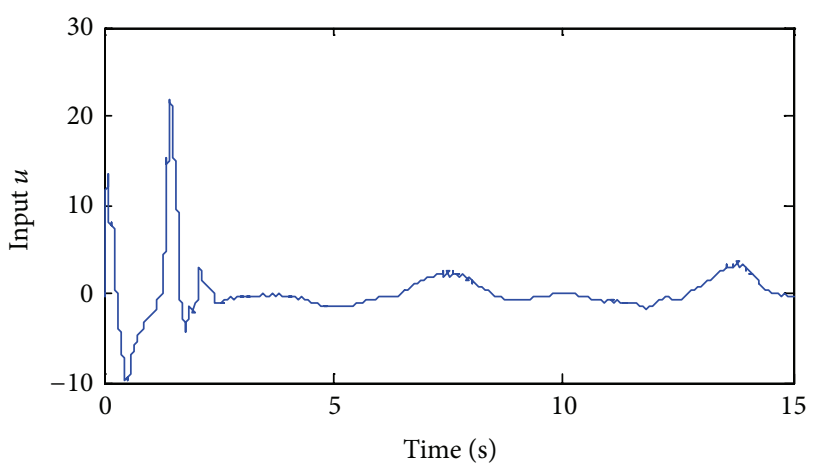

FIgUre 10: Actual input $u$.

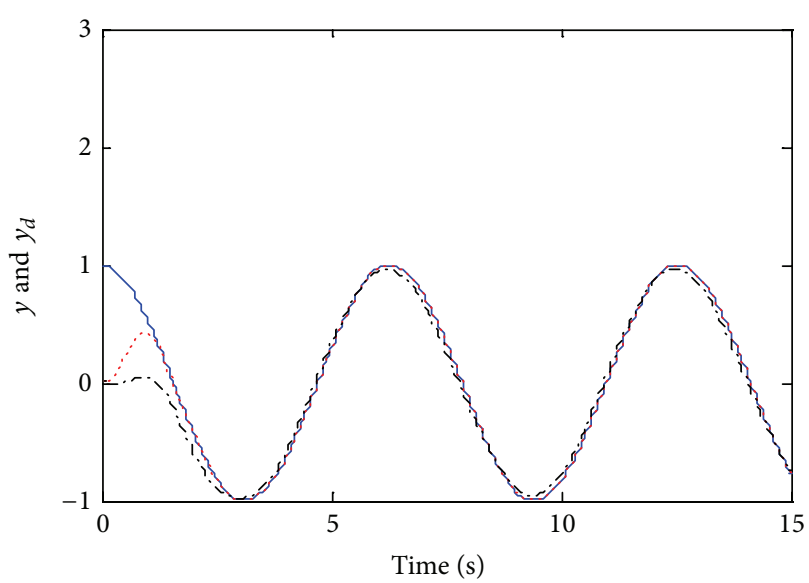

$-y_{d}$

..... Proposed control

.... Backstepping-like control

FIGURE 11: Tracking performance of the two controllers.

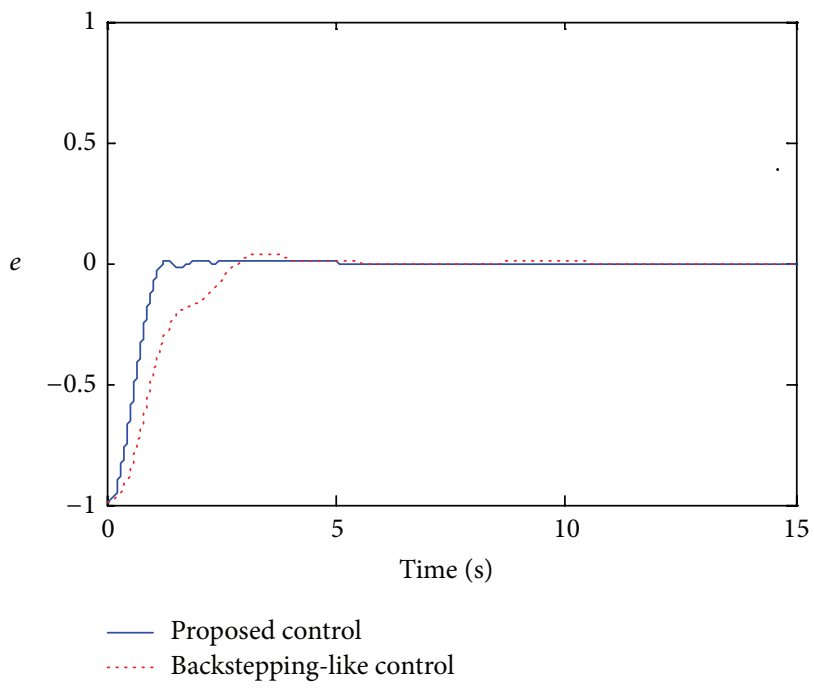

FIGURE 12: Tracking error of the two controllers.

According to the simulation results, the following can be concluded:

(1) The proposed controller can insure the tracking error and its derivatives have a finite-time convergence property (Figures $2-5$ ). This is because the proposed controller can insure a finite-time convergence of the sliding mode surface (Figure 6); moreover, the sliding mode surface in this paper is a nonsingular finitetime terminal sliding mode surface, so the states on the sliding mode surface can converge to zero in finite time.

(2) The proposed sliding mode controller is chatteringfree. Though the augmented input is desecrated (Figure 9), the actual input $u$ is continuous and smooth, because $u$ is produced by $\dot{u}=T u+v$. 
Thus, the chattering of the conventional sliding mode control is avoided.

(3) The proposed controller is simple and easy to implement. And the complexity of the current controllers designed for nonaffine systems is greatly avoided. It is noted that three NN units, two filters, and all the three states are needed in the backstepping-like controller proposed in [18]. And the number of NN units and filters will increase with the increase of the system order. However, only one output state, one differentiator, and one filter are needed in the controller proposed in this paper. In addition, the number of the needed states, differentiators, and filters will not increase with the increase of the system order. So the complexity of the controller for nonaffine pure-feedback systems is greatly avoided.

(4) Compared to the controller in [18], the needed knowledge of system is reduced. In the proposed controller, only the output and the order of the system, other than all the states and the accurate system model, are needed.

(5) According to Figures 11-12, it is shown that the control performance of the proposed controller in this paper is as good as that of the improved backstepping-like controller proposed in [18].

\section{Conclusion}

In the paper, a novel control scheme with low complexity is proposed for unknown nonaffine pure-feedback systems. The scheme involves a novel transformed method, which can transform the nonaffine systems into affine strict-feedback system. And a nonsingular finite-time sliding mode controller is also implemented. Compared with the related article, the proposed scheme not only can achieve finite-time convergence, but also is much simpler and easy to implement and needs less system knowledge. And the simulation results demonstrate that the tracking performance is also very good.

\section{Conflict of Interests}

The authors declare that there is no conflict of interests regarding the publication of this paper.

\section{Acknowledgments}

This work is supported by the National Natural Science Foundation of China (Grant no. 61304238) and the National HighTech R\&D Program of China (Grant no. 2012AA120602).

\section{References}

[1] V. Isidori, Nonlinear Control Systems, Springer, New York, NY, USA, 1989.

[2] M. Kristic, I. Kanellakopoulos, and P. V. Kokoyovic, Nonlinear and Adaptive Control Design, Wiley, New York, NY, USA, 1995.
[3] K. P. Tee, S. S. Ge, and F. E. H. Tay, "Adaptive neural network control for helicopters in vertical flight," IEEE Transactions on Control Systems Technology, vol. 16, no. 4, pp. 753-762, 2008.

[4] H. Wang, X. Yang, Z. Yu, K. Liu, and X. Liu, "Fuzzyapproximation-based decentralized adaptive control for purefeedback large-scale nonlinear systems with time-delay," Neural Computing and Applications, vol. 26, no. 1, pp. 151-160, 2015.

[5] J. Zhang, Q. Zhu, X. Wu, and Y. Li, "A generalized indirect adaptive neural networks backstepping control procedure for a class of non-affine nonlinear systems with pure-feedback prototype," Neurocomputing, vol. 121, no. 9, pp. 131-139, 2013.

[6] C. Wang, D. J. Hill, S. S. Ge, and G. Chen, "An ISS-modular approach for adaptive neural control of pure-feedback systems," Automatica, vol. 42, no. 5, pp. 723-731, 2006.

[7] S. S. Ge and C. Wang, "Direct adaptive NN control of a class of nonlinear systems," IEEE Transactions on Neural Networks, vol. 13, no. 1, pp. 214-221, 2002.

[8] S. S. Ge and C. Wang, "Adaptive NN control of uncertain nonlinear pure-feedback systems," Automatica, vol. 38, no. 4, pp. 671-682, 2002.

[9] D. Wang and J. Huang, "Adaptive neural network control for a class of uncertain nonlinear systems in pure-feedback form," Automatica, vol. 38, no. 8, pp. 1365-1372, 2002.

[10] J. Wen and C. Jiang, "Adaptive fuzzy controller for a class of strict-feedback nonaffine nonlinear systems," Journal of Systems Engineering and Electronics, vol. 22, no. 6, pp. 967-974, 2011.

[11] Y. Gao, S. Tong, and Y. Li, "Adaptive fuzzy backstepping output feedback control for a class of uncertain stochastic nonlinear system in pure-feedback form," Neurocomputing, vol. 122, no. 25, pp. 126-133, 2013.

[12] Y.-J. Liu and W. Wang, "Adaptive fuzzy control for a class of uncertain nonaffine nonlinear systems," Information Sciences, vol. 177, no. 18, pp. 3901-3917, 2007.

[13] J. Wen and C.-S. Jiang, "Adaptive fuzzy control for a class of chaotic systems with nonaffine inputs," Communications in Nonlinear Science and Numerical Simulation, vol. 16, no. 1, pp. 475-492, 2011.

[14] H. Du, H. Shao, and P. Yao, "Adaptive neural network control for a class of low-triangular-structured nonlinear systems," IEEE Transactions on Neural Networks, vol. 17, no. 2, pp. 509-514, 2006.

[15] P. P. Yip and J. K. Hedrick, "Adaptive dynamic surface control: a simplified algorithm for adaptive backstepping control of nonlinear systems," International Journal of Control, vol. 71, no. 5, pp. 959-979, 1998.

[16] D. Swaroop, J. K. Hedrick, P. P. Yip, and J. C. Gerdes, "Dynamic surface control for a class of nonlinear systems," IEEE Transactions on Automatic Control, vol. 45, no. 10, pp. 1893-1899, 2000.

[17] T. P. Zhang and S. S. Ge, "Adaptive dynamic surface control of nonlinear systems with unknown dead zone in pure feedback form," Automatica, vol. 44, no. 7, pp. 1895-1903, 2008.

[18] G. Sun, D. Wang, X. Li, and Z. Peng, "A DSC approach to adaptive neural network tracking control for pure-feedback nonlinear systems," Applied Mathematics and Computation, vol. 219, no. 11, pp. 6224-6235, 2013.

[19] C. P. Bechlioulis and G. A. Rovithakis, "A low-complexity global approximation-free control scheme with prescribed performance for unknown pure feedback systems," Automatica, vol. 50, no. 4, pp. 1217-1226, 2014.

[20] A. Šabanovic, "Variable structure systems with sliding modes in motion control-a survey," IEEE Transactions on Industrial Informatics, vol. 7, no. 2, pp. 212-223, 2011. 
[21] Y. Feng, F. Han, and X. Yu, "Chattering free full-order slidingmode control," Automatica, vol. 50, no. 4, pp. 1310-1314, 2014.

[22] M. Zhihong and X. H. Yu, "Terminal sliding mode control of MIMO linear systems," IEEE Transactions on Circuits and Systems I: Fundamental Theory and Applications, vol. 44, no. 11, pp. 1065-1070, 1997.

[23] Y. Wu, X. Yu, and Z. Man, "Terminal sliding mode control design for uncertain dynamic systems," Systems and Control Letters, vol. 34, no. 5, pp. 281-287, 1998.

[24] Y. Feng, X. Yu, and Z. Man, "Non-singular terminal sliding mode control of rigid manipulators," Automatica, vol. 38, no. 12, pp. 2159-2167, 2002.

[25] L. Yang and J. Yang, "Nonsingular fast terminal sliding-mode control for nonlinear dynamical systems," International Journal of Robust and Nonlinear Control, vol. 21, no. 16, pp. 1865-1879, 2011.

[26] H. Sun, S. Li, and C. Sun, "Finite time integral sliding mode control of hypersonic vehicles," Nonlinear Dynamics, vol. 73, no. 1-2, pp. 229-244, 2013.

[27] M.-J. Zhang and Z.-Z. Chu, "Adaptive sliding mode control based on local recurrent neural networks for underwater robot," Ocean Engineering, vol. 45, no. 1, pp. 56-62, 2012.

[28] G. S. Tombul, S. P. Banks, and N. Akturk, "Sliding mode control for a class of non-affine nonlinear systems," Nonlinear Analysis: Theory, Methods \& Applications, vol. 71, no. 12, pp. e1589-e1597, 2009.

[29] G. Bartolini and E. Punta, "Sliding mode output-feedback stabilization of uncertain nonlinear nonaffine systems," Automatica, vol. 48, no. 12, pp. 3106-3113, 2012.

[30] H. M. Gutierrez and P. I. Ro, "Magnetic servo levitation by sliding-mode control of nonaffine systems with algebraic input invertibility," IEEE Transactions on Industrial Electronics, vol. 52, no. 5, pp. 1449-1455, 2005.

[31] G. Bartolini and E. Punta, "Reduced-order observer in the sliding-mode control of nonlinear nonaffine systems," IEEE Transactions on Automatic Control, vol. 55, no. 10, pp. 23682373, 2010.

[32] G. Bartolini and E. Punta, "Multi-input sliding mode control of nonlinear uncertain non-affine systems with mono-directional actuation," IEEE Transactions on Automatic Control, vol. 60, no. 2, pp. 393-403, 2015.

[33] Y. Hong, J. Huang, and Y. Xu, "On an output feedback finitetime stabilization problem," IEEE Transactions on Automatic Control, vol. 46, no. 2, pp. 305-309, 2001.

[34] S. P. Bhat and D. S. Bernstein, "Continuous finite-time stabilization of the translational and rotational double integrators," IEEE Transactions on Automatic Control, vol. 43, no. 5, pp. 678-682, 1998.

[35] J. Yang, J. Su, S. Li, and X. Yu, "High-order mismatched disturbance compensation for motion control systems via a continuous dynamic sliding-mode approach," IEEE Transactions on Industrial Informatics, vol. 10, no. 1, pp. 604-614, 2014.

[36] W. J. Rugh, Linear System Theory, Johns Hopkins University Press, Baltimore, Md, USA, 2002. 


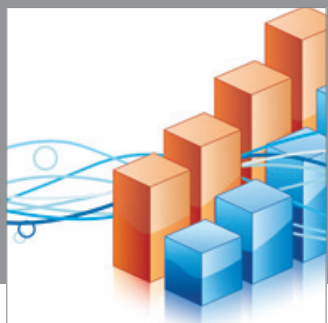

Advances in

Operations Research

mansans

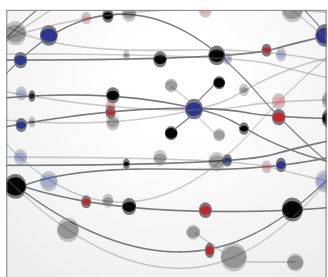

The Scientific World Journal
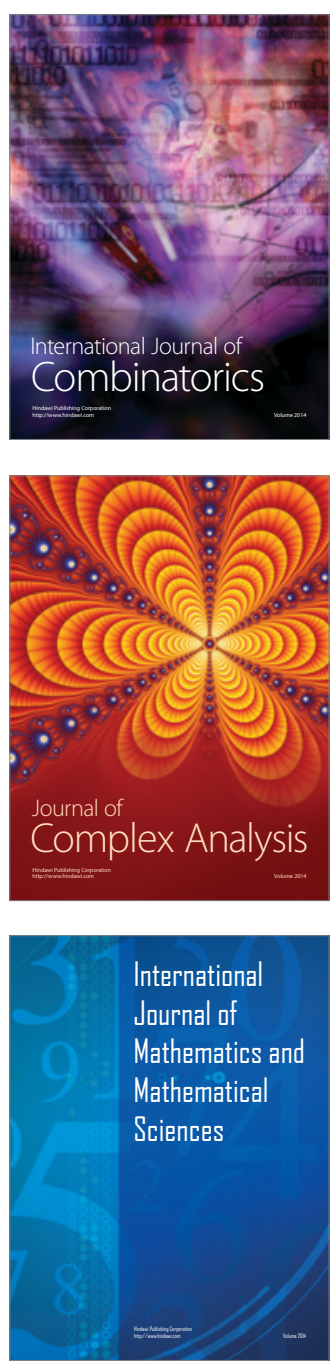
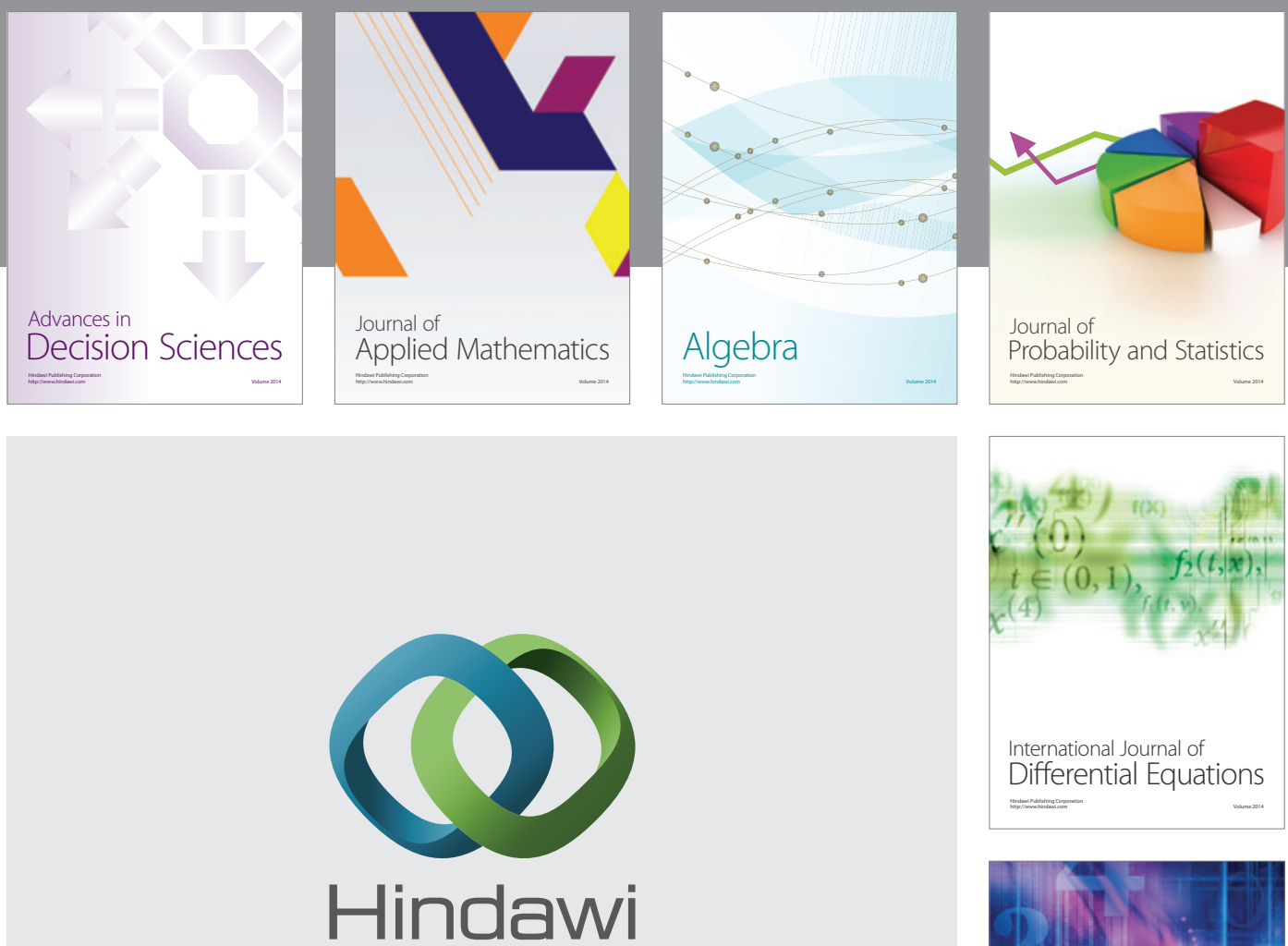

Submit your manuscripts at http://www.hindawi.com
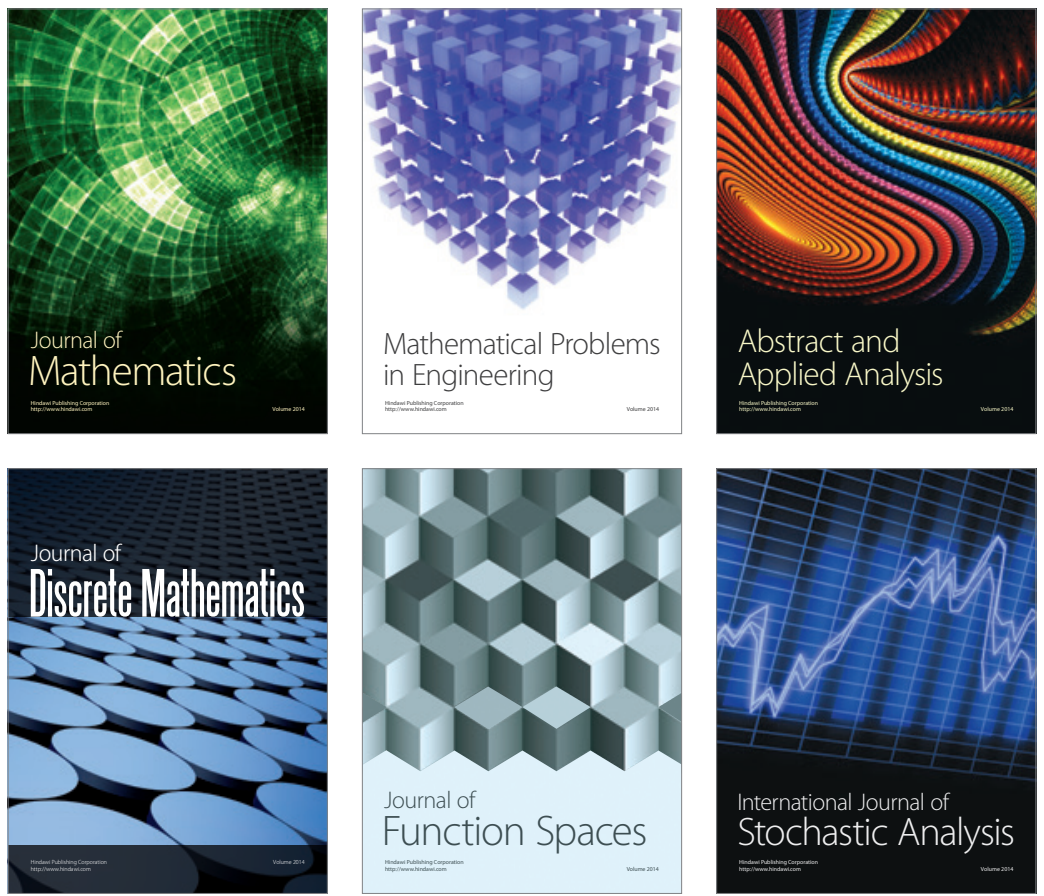

Journal of

Function Spaces

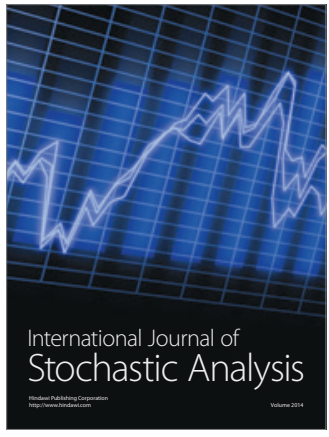

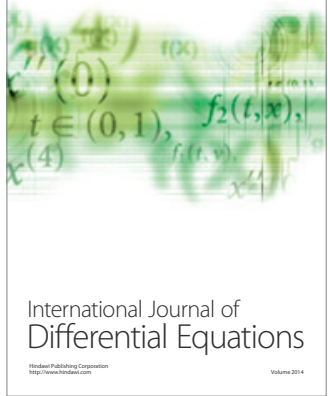
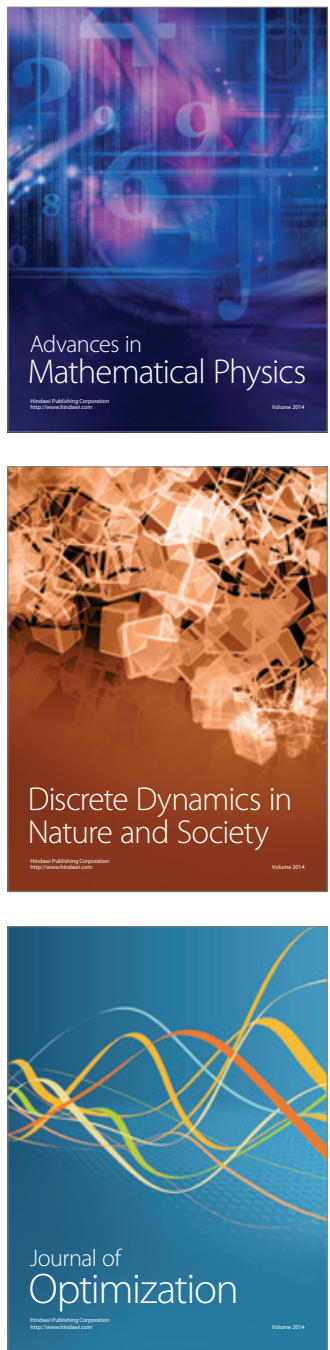